\title{
Congestion Allocation for Distributed Networks: An Experimental Study *
}

December 16, 2006

Yan Chen, School of Information, University of Michigan, 1075 Beal Avenue, Ann Arbor, MI 48109-2112. Email: yanchen@umich.edu.

Laura Razzolini Department of Economics, School of Business, Virginia Commonwealth University, 1015 Floyd Avenue, Box 844000 Richmond, VA 23284-4000.

E-mail: lrazzolini@vcu.edu.

Theodore L. Turocy Department of Economics, Texas A\&M University, College Station TX 77843.

Email: turocy@econmail.tamu.edu

Abstract This paper reports an experimental study of two prominent congestion and cost allocation mechanisms for distributed networks. We study the fair queuing (or serial) and the FIFO (or average cost pricing) mechanisms under two different treatments: a complete information treatment and a limited information treatment designed to simulate distributed networks. Experimental results show that the fair queuing mechanism performs significantly better than FIFO in all treatments in terms of efficiency, predictability measured as frequency of equilibrium play, and the speed of convergence to equilibrium.

Keywords serial cost sharing · congestion allocation · experiment

JEL Classification Numbers C91 · D83

\footnotetext{
*We thank Simon Anderson, Narine Badasyan, Catherine Eckel, Peter Katuscak, Edna Lohman, seminar participants at Caltech, Michigan, Vanderbilt, Virginia, Virginia Tech, ESA 2001 (Tucson, AZ) and Public Choice Society meetings (2002) for helpful comments. We are grateful to David Cooper and an anonymous referee for their constructive and insightful suggestions which significantly improved the paper. We thank Jing Deng, Belal Sabki, Pragya Sen, Brian Chan and Lisa Robinson for excellent research assistance, and Jim Leady for programming for the experiment. The research support provided by NSF grant SES-0079001 to Chen, SES-9973731 to Razzolini, and a grant from the Office of Naval Research to Razzolini are gratefully acknowledged. Any remaining errors are our own.
} 


\section{Introduction}

There has been much interest in the problem of congestion allocation in computer networks, as the Internet becomes increasingly important in global telecommunications and e-commerce. In distributed networks such as the Internet, multiple agents share the same network link. Each agent controls the rate at which she is transmitting data. If the sum of the transmission rates is greater than the total link capacity, then the link becomes congested and the agents' packets experience delays. Most current Internet routers use a FIFO packet scheduling algorithm, where all packets are serviced on a first-come-first-serve basis. Each user's average queue is proportional to their throughput (Shenker 1990). One agent's usage can affect the quality of service of other agents. Aggressive users can get more than an equal share of these shared facilities. For example, agents who modify their Transmission Control Protocol implementation to be less responsive when congestion is detected can obtain much larger shares of the bandwidth (Demers and Shenker 1990). In contrast, the Fair Queuing packet scheduling algorithm, originally proposed by (Shenker 1990), leads to congestion allocations such that an agent's average queue is independent of transmission rates higher than her own. For example, if each user contributes an independent Poisson input stream of packets with various rates, the Fair Queuing algorithm allocates congestion by a preemptive priority queuing algorithm, where users are ordered by increasing transmission rates. All of the smallest user's packets are in the highest priority class, and all of the other users get the same rate (as the smallest user) of packets in the highest priority class. Similarly, the rest of the second smallest user's packets are in the second highest priority class, and all of the other users gets the same rate of packets in the second highest priority class; and so on. The Fair Queuing algorithm has been proposed as an alternative to the FIFO algorithm, based on theoretical and simulation results (Stoica et al. 1998). The new generation of Cisco 7200, 3600 and 2600 routers have both the FIFO and Fair Queuing options. In this paper, we evaluate the performance of these two algorithms using laboratory experiments.

Congestion allocation in distributed networks is closely related to the more general class of cost sharing

problems. A cost-sharing mechanism distributes the service and allocates the corresponding costs to each agent. The FIFO packet scheduling algorithm corresponds to the average cost pricing mechanism (Shenker 1990), where an agent's cost share is proportional to her own demand, while the Fair Queuing algorithm corresponds to the serial cost sharing mechanism.

The theoretical literature on cost sharing has largely focused on the axiomatic characterization of these mechanisms (e.g., Moulin and Shenker (1994); Friedman and Moulin (1999)) and their static properties in a complete information setting with synchronous actions. However, as Friedman and Shenker (1998) 
point out, in a distributed system ${ }^{1}$ such as the Internet where agents have very limited a priori information about other agents and the payoff structure, traditional solution concepts might not be able to predict the outcome of learning. It is important to empirically study the actual learning dynamics among real players in settings similar to distributed networks and examine whether learning will lead to the equilibrium predicted by theory. This paper does this by investigating the learning dynamics induced by each mechanism under both complete and limited information settings in a laboratory environment.

We are aware of few other experimental studies of cost sharing mechanisms: Chen (2003) and Razzolini et al. (1999). ${ }^{2}$ Chen (2003) studies the serial and average cost pricing mechanisms under complete and limited information with only two types of agents. Razzolini et al. (1999), on the other hand, investigate the performance of the serial mechanism with four players: one human and three computerized. The human player knows his own cost share and payoff structure but has no information about the opponents' payoff structures. This information condition is in between the complete information and the limited information setting in Chen (2003). Chen (2003) found that the performance of the two mechanisms is statistically indistinguishable under complete information. Under limited information, however, the serial mechanism performs robustly better than the average cost pricing mechanism in terms of frequency of equilibrium play and system efficiency. ${ }^{3}$ Razzolini et al. (1999) implement the serial mechanism both as a sequential and a simultaneous normal form game. They also found that the serial mechanism leads to almost efficient allocations, and, even though more easy to understand and implement, the simultaneous move treatment does not lead to a better overall performance.

In this paper, we design an experiment to evaluate the serial and the average cost pricing mechanisms in a baseline complete information environment, and in a more challenging environment with limited information. In our experiment, each session has twelve players of four different types. This environment is, therefore, much more complex than the one in the two earlier studies. Chen (2003) can use a payoff table to explain both mechanisms, which is feasible for the serial mechanism with only two types of players. When the number of types increases, the serial mechanism becomes more challenging to implement in

\footnotetext{
${ }^{1}$ Following Friedman and Shenker (1998), a system is called a distributed system "because the users are geographically dispersed and are accessing the resource through the network." The Internet is a prominent example.

${ }^{2}$ In addition, Gailmard and Palfrey (2005) report experiments for the provision of excludable threshold public goods and compare the serial cost sharing mechanism with voluntary cost sharing with proportional rebates and with no rebates. Rapoport et al. (2004) report an experimental study of a large-scale queuing game with the FIFO queue discipline (i.e., average cost sharing mechanism).

${ }^{3}$ Chen and Khoroshilov (2003) study the learning dynamics in these cost sharing games and other games under limited information.
} 
the laboratory, because the dimension of the payoff tables increases with each additional type. With more than two types one needs to find alternative ways to implement the mechanism. Razzolini et al. (1999) has four different types, but only one of them is a human player, thus the strategic interaction between types is simplified.

While the previous studies present the first steps in understanding how these mechanisms work, the goal of this paper is to assess the performance of the two mechanisms in different settings and with more than just two types; to study how human subjects learn in these different settings; whether and how the learning dynamics leads to convergence to stage game Nash equilibrium; and ultimately test the practical implementability of the fair queuing or serial mechanism in comparison to the average cost pricing mechanism. The earlier studies considered special cases, only two subjects or one human and three automated players, which made it possible for the serial mechanism to be presented to subjects in a very simple way. The environment considered in this paper with four different types interacting is more realistic and complex, and forced us to change the way the mechanism is presented to the subjects. In contrast with previous studies, we find that under both complete and limited information settings the serial mechanism performs robustly better than average cost pricing in terms of the proportion of equilibrium play, speed of convergence and efficiency. The more complex environment considered in this paper changed the relative performance of the two mechanisms with respect to previous studies, with the serial mechanism uniformly outperforming the average cost pricing. This is mostly due to the strategic properties of the serial mechanism, which facilitate learning and convergence.

The paper is organized as follows. Section 2 introduces the theoretical properties of the serial (hereafter shortened as SRL) and average cost pricing (hereafter shortened as ACP) mechanisms. Section 3 presents the experimental design. Section 4 compares the performance of the mechanisms under complete information and limited information. Section 5 concludes the paper.

\section{Theoretical Properties of the Mechanisms}

Let $N=\{1, \cdots, i, \cdots, n\}$ be a group of agents sharing a one-input, one-output technology. Each of the $n$ agents announces her demand $q_{i}$ of output. Each agent gets her demand $q_{i}$ and pays a cost share, $x_{i}$. Note $x_{i}$ is the total cost agent $i$ pays. In the example of Internet routers, $q_{i}$ is agent $i$ 's data transmission rate, while $x_{i}$ is the reduction in agent $i$ 's utility due to congestion. Let $q_{1} \leq q_{2} \leq \cdots \leq q_{n}$. The cost function is denoted by $C$, which is strictly convex. A cost-sharing mechanism must allocate the total $\operatorname{cost} C\left(\sum_{i} q_{i}\right)$ among the $n$ agents. 
The serial mechanism, originally introduced by Shenker (1990), was analyzed by Moulin and Shenker (1992) in the context of cost and surplus sharing with complete information. The mechanism can be characterized by four properties: unique Nash equilibrium at all profiles ${ }^{4}$, anonymity (the name of the agents does not matter), monotonicity (an agent's cost share increases when she demands more output) and smoothness (an agent's cost share is a continuously differentiable function of the vector of demands). Among agents endowed with convex, continuous and monotonic preferences, the serial mechanism is the only cost sharing rule which is dominance-solvable, and its unique Nash equilibrium is also robust to coalitional deviations when agents cannot transfer outputs.

Under the serial mechanism, agent 1 (with the lowest demand) pays $(1 / n)$ th of the cost of producing $n q_{1}, x_{1}^{s}=C\left(n q_{1}\right) / n$. Agent 2 pays agent 1's cost share plus $1 /(n-1)$ th of the incremental cost from $n q_{1}$ to $(n-1) q_{2}+q_{1}$, i.e.,

$$
x_{2}^{s}=\frac{C\left(n q_{1}\right)}{n}+\frac{C\left(q_{1}+(n-1) q_{2}\right)-C\left(n q_{1}\right)}{n-1},
$$

and so on. Let $q^{0}=0 ; \quad q^{1}=n q_{1} ; \quad q^{2}=q_{1}+(n-1) q_{2} ; \cdots ; \quad q^{i}=q_{1}+\cdots+q_{i-1}+(n+1-i) q_{i} ; \cdots, \quad q^{n}=$ $\sum_{i} q_{i}$. Then the general formula for agent $i$ 's cost share is given by

$$
x_{i}^{s}(c, q)=\sum_{k=1}^{i} \frac{C\left(q^{k}\right)-C\left(q^{k-1}\right)}{n+1-k}, \text { for all } i=1, \cdots, n .
$$

Therefore, an agent's cost share under the serial mechanism is only affected by her own demand and those whose demands are lower than hers. An agent's cost share is independent of demands higher than her own.

Like the serial mechanism, the average cost pricing mechanism satisfies anonymity, monotonicity and smoothness. It is the only method that is robust to arbitrage, i.e., agents cannot benefit from merging or splitting their demands. In contrast to the serial mechanism, the normal form game induced by the average cost pricing mechanism is in general not dominance-solvable, nor does it have a unique equilibrium at all profiles when agents have convex, continuous and monotonic preferences.

When agent $i$ demands $q_{i}$ amount of output, the general formula for agent $i$ 's cost share under the average cost pricing mechanism is given by

$$
x_{i}^{a}(c, q)=\left(q_{i} / \sum_{k} q_{k}\right) \cdot C\left(\sum_{k} q_{k}\right), \text { for all } i=1, \cdots, n .
$$

Therefore, under ACP an agent's cost share is determined by the proportion of the total demand for which her own demand accounts. It is affected by her own demand, and the sum of all other agents' demands.

There is no systematic efficiency comparison between the two mechanisms. In general there exists no differentiable and monotonic cost sharing mechanism where all Nash equilibrium outcomes are first best

\footnotetext{
${ }^{4}$ Assume agents have convex, continuous and monotonic preferences.
} 
Pareto optimal at all preference profiles. Moulin and Shenker (1992) provide a definition of second best efficiency ${ }^{5}$ and show that the serial mechanism yields a second best efficient equilibrium while ACP does not.

In distributed systems, such as the Internet where users are geographically dispersed and have little information about other players and the payoff structure, for learning to converge to equilibrium, it is important that strategies sampled by players are informative. In this respect, the serial mechanism has an advantage over ACP.

We first summarize several relevant concepts. In a normal form game, one action overwhelms another if all payoffs, over all sets of other players' actions, for the one are greater than all payoffs, over all sets of other players' actions, for the other action. The serially unoverwhelmed set is the set remaining after iterated elimination of overwhelmed actions. A game is $D$-solvable if iterated elimination of dominated strategies leads to a single eventual outcome. A game is $O$-solvable if iterated elimination of overwhelmed strategies leads to a single eventual outcome. Therefore, overwhelming implies dominance, and thus, o-solvability implies d-solvability. Another name for overwhelming is uniform dominance. Huck and Sarin (2004) prove that, for the class of uniformly dominance solvable games, i.e., O-solvable games, the unique Nash equilibrium is a stable limited memory equilibrium regardless of evaluation rules and memory capacities. Friedman and Shenker (1998) prove that reasonable learners ${ }^{6}$ converge to the serially unoverwhelmed set. In comparison, Milgrom and Roberts (1990) showed that adaptive learners converge to the serially undominated set.

Among the cost sharing mechanisms studied in this paper, the serial mechanism is O-solvable ${ }^{7}$ while ACP is not. That is, in the serial game iterated elimination of overwhelmed strategies leads to a single eventual outcome. When a strategy overwhelms another one, sampling is much more informative than situations where one strategy dominates another, as the minimum payoff from the overwhelming strategy is at least as large as the maximum payoff of the overwhelmed strategy. In environments with limited information, such as the Internet, informative sampling can significantly increase the speed of learning.

\footnotetext{
5"For an arbitrary cost sharing mechanism $\xi$, say that $\left(q_{1}, \cdots, q_{n}\right)$ is a Nash equilibrium outcome at some utility profile. We ask if there is another vector of demands $\left(q_{1}^{\prime}, \cdots, q_{n}^{\prime}\right)$ such that at the corresponding allocation dictated by the mechanism $\xi$, no one is worse off and someone is better off than at the equilibrium allocation corresponding to $\left(q_{1}, \cdots, q_{n}\right)$. If no such vector of demands exists, we call our equilibrium second best efficient.” Moulin and Shenker (1992, p.1025)

${ }^{6}$ The key components of a reasonable learner are optimization, monotonicity and responsiveness (Friedman and Shenker 1998).

${ }^{7}$ This is proved in Theorem 8 in Friedman and Shenker (1998).
} 


\section{Experimental Design}

The goal of the experimental design is to compare the performance of the SRL and ACP mechanisms in two different settings: a complete information setting that tests the prediction of dominance-solvability, and a more challenging network setting to compare the performance of the two mechanisms and to assess the plausibility of the concept of uniform dominance. The economic environment and experimental procedures are discussed in the sections below.

\subsection{Experimental Procedures}

We implemented a $2 \times 2$ factorial design by varying the mechanisms and information conditions. We conducted five independent sessions for each of the four treatments. Each session had twelve subjects of four different types and last for fifty rounds. Players always kept their own type. For a baseline comparison, we conducted ten sessions of the SRL and ACP mechanisms under complete information with the random matching protocol (hereafter shortened as $\mathrm{SRL}_{c}$ and $\mathrm{ACP}_{c}$ ). Under complete information, each player was informed of the structure of the game, matching protocols, the quantities chosen and the corresponding payoffs earned by all players in all rounds. This pair of treatments were designed to compare the performance of the two mechanisms as one-shot games under complete information. To evaluate the possibility of applying these mechanisms to distributed systems such as the Internet, we designed a pair of limited information treatments. Learning in distributed systems is characterized by the feature that players might have extremely limited information. They often do not know the payoff functions, nor do they know how their payoffs depend on the actions of others, probably due to the lack of information about the detailed nature of the resources itself. Therefore, in the limited information treatments, the only information players had was their own action and the resulting own payoffs. In these treatments, players were again randomly re-matched into groups of four in each round (hereafter shortened as $\mathrm{SRL}_{l}$ and $\mathrm{ACP}_{l}$ ). Table 1 summarizes features of the experimental design.

[Table 1 about here. ]

Computerized experiments were conducted at the RCGD Laboratory at the University of Michigan in July and August, 2001. We used z-Tree (Fischbacher 1999) to program our experiments. We conducted twenty independent sessions. Subjects were students from the University of Michigan. A total of 240 subjects participated in the experiment. No subject was used in more than one session. 
At the beginning of each session subjects randomly drew a PC terminal number. Then each of them was seated in front of the corresponding terminal, and given the instructions. After the instructions were read aloud, subjects were required to finish the Review Questions in the complete information treatment, which were designed to test their understanding of the instructions. Since the instructions for the limited information case were straightforward, those subjects were not given Review Questions. Afterwards the experimenter checked answers and answered questions. In all complete information sessions the instruction period was within 25 minutes and the entire session took about one hour. In all limited information sessions the instruction period was within 10 minutes and the entire session took approximately 40 minutes. There was no practice round in any session. The average earning was $\$ 19.03$, including a $\$ 3$ show-up fee.

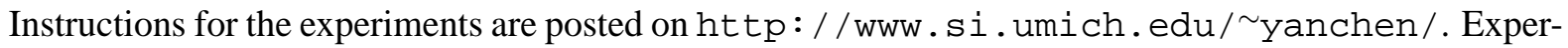
imental data are available from the authors upon request.

\subsection{The Economic Environment}

Each of the four types $i=1,2,3,4$ are endowed with linear preferences $\pi_{i}\left(x_{i}, q\right)=\alpha_{i} q_{i}+\omega_{i}-x_{i}$, where $\alpha_{i}$ is agent $i$ 's marginal utility for the output, $\omega_{i}$ is agent $i$ 's lump-sum endowment and $x_{i}$ is her cost share. The cost function is chosen to be quadratic, $C(q)=q^{2}$. We call this environment $E$. In the network context with several agents sharing a network link, $\alpha_{i}$ is agent $i$ 's value for the amount of data transmitted per unit of time, and the cost to be allocated corresponds to the congestion experienced. Therefore, the cost should be interpreted as the reduction in agent $i$ 's utility due to congestion. The types are indexed in order of increasing marginal utility for the output, $\alpha_{1} \leq \alpha_{2} \leq \alpha_{3} \leq \alpha_{4}$; in the instructions, we refer to player 1 as Blue, player 2 as Green, player 3 as Red, and player 4 as Yellow. The values of these parameters used in the experimental sessions are reported in Table 2.

[Table 2 about here.]

Under the serial mechanism, the cost share for agent 1 is $x_{1}^{s}=C\left(4 q_{1}\right) / 4$. Agent 2's cost share is $x_{2}^{s}=x_{1}^{s}+\left(C\left(q_{1}+3 q_{2}\right)-C\left(4 q_{1}\right)\right) / 3$. Agent 3's cost share is $x_{3}^{s}=x_{2}^{s}+\left(C\left(q_{1}+q_{2}+2 q_{3}\right)-C\left(q_{1}+3 q_{2}\right)\right) / 2$. Agent 4's cost share is $x_{4}^{s}=x_{3}^{s}+\left(C\left(q_{1}+q_{2}+q_{3}+q_{4}\right)-C\left(q_{1}+q_{2}+2 q_{3}\right)\right)$. For the ACP mechanism,

the cost shares of each of the four agents are $x_{i}^{a}=\frac{q_{i}}{\sum_{i=1}^{4} q_{i}} C\left(\sum_{i=1}^{4} q_{i}\right)=q_{i}\left(\sum_{i=1}^{4} q_{i}\right)$. In our design, the SRL and ACP mechanisms are implemented as normal form games with a discrete strategy space for each player, $\{0,1, \cdots, 19,20\}$.

Using Gambit (McKelvey et al. 2006), we find that it takes five steps of iterated dominance to compute the unique equilibrium of the SRL game: 
Step 1. No strategy overwhelms another. After elimination of dominated strategies, $\{\{6,7, \cdots, 20\},\{7,8$, $\cdots, 20\},\{7,8, \cdots, 20\},\{7,8, \cdots, 20\}\}$ remain.

Step 2. For player 1, 6 overwhelms every other strategy, while for player 2, 7 (weakly) overwhelms every other strategy. After elimination of overwhelmed and dominated strategies, $\{\{6\},\{7\},\{7,8,9\},\{7$, $8,9,10\}$ \} remain undominated.

Step 3. No strategy overwhelms another. The remaining undominated strategies are $\{\{6\},\{7\},\{8,9\},\{8,9$, $10\}\}$.

Step 4. For player 3, 8 overwhelms 9, while for player 4, 9 (weakly) overwhelms 10. The undominated strategies are $\{\{6\},\{7\},\{8\},\{8,9\}\}$.

Step 5. For player 4, 9 dominates 8 , leaving $\{\{6\},\{7\},\{8\},\{9\}\}$ as the D-solvable equilibrium.

The unique equilibrium $(6,7,8,9)$ of the SRL game remains the unique equilibrium of the game if agents are permitted to choose from a continuum of demands. The equilibrium in the continuous version is both D-solvable and O-solvable.

OBSERVATION 1 In environment $E$ under the SRL mechanism, if $\left\{q_{i}^{*}\right\}_{i \in N}$ is the unique Nash equilibrium of the continuous game, it remains the unique Nash equilibrium of the discrete game. Furthermore, the $O$ solvable equilibrium with a continuous strategy space is D-solvable with a discrete strategy space.

With the discrete strategy space, no strategies in the ACP game are dominated. There are a total of 19 equilibria, all of which are in pure strategies. One equilibrium, $(4,10,14,16)$, is the unique, D-solvable equilibrium of the ACP game with continuous demands, and is the only strict Nash equilibrium of the discrete game. Table 3 lists all nineteen pure strategy Nash equilibria for the ACP game. They are organized by the equilibrium quantities from the smallest demander to the largest demander. Note all 19 equilibria have the same aggregate demand, $\sum_{i=1}^{4} q_{i}^{a}=44$. The last column lists the aggregate equilibrium payoffs in decreasing order.

\section{[Table 3 about here.]}

Multiple equilibria as a result of discretization is a generic property of the average cost pricing mechanism, regardless of the step size for discretization. Let $D$ be a discrete strategy space such that the equilibrium of the continuous strategy space, $\left\{q_{i}^{*}\right\}_{i \in N} \in D$. Let $s>0$ be the step size in $D$. The following propositions characterize the Nash equilibria of the ACP mechanism with a discrete strategy space $D$. 
PROPOSITION 1 In environment $E$ under the ACP mechanism, if $\left\{q_{i}^{*}\right\}_{i \in N}$ is the unique Nash equilibrium of the continuous game, then the set of pure strategy equilibria in the discretized game is $\left\{\bar{q}_{1}, \cdots, \bar{q}_{n} \mid \bar{q}_{i} \in\right.$ $\left\{q_{i}^{*}-s, q_{i}^{*}, q_{i}^{*}+s\right\}$ and $\left.\sum_{i} \bar{q}_{i}=\sum_{i} q_{i}^{*}, \forall i \in N, \forall s>0\right\}$.

Proof: see Appendix.

PROPOSITION 2 In environment E under the ACP mechanism, there does not exist any mixed strategy equilibria.

Proof: see Appendix.

Based on the theoretical properties of the mechanisms and the design, we formally state the following null hypotheses.

HYPOTHESIS 1 Under complete information, SRL and ACP will generate the same proportion of equilibrium play.

HYPOTHESIS 2 Under limited information, SRL and ACP will generate the same proportion of equilibrium play.

While we expect the performance of the two mechanisms to be the same under complete information, under limited information, however, as much the dominance (although not all) is also uniform dominance, we expect SRL to have higher level of equilibrium play.

HYPOTHESIS 3 Under complete information, the speed of convergence is the same under SRL and ACP.

HYPOTHESIS 4 Under limited information, the speed of convergence is the same under SRL and ACP.

Again, while we expect the speed of convergence to be the same under complete information, we expect convergence to be more rapid under SRL under limited information.

We next define an efficiency measure for the mechanisms. In general, there is no systematic efficiency comparison between the two mechanisms, as it is possible to find preference profiles, such that either the SRL game or the ACP game can yield higher aggregate payoffs. In the design, we give each player a lumpsum payment, so that the equilibrium aggregate payoffs under the two mechanisms are the same as with a continuous strategy space. Following the convention in the experimental economics literature, we define efficiency as the ratio of the sum of the actual earnings of all subjects in a session and the maximum possible 
aggregate earnings. ${ }^{8}$ As a benchmark, if players play their Nash equilibrium strategies, the efficiency is $96 \%$ for SRL and between $94 \%$ and $98 \%$ for ACP.

\section{Results}

In this section, we compare the performance of the two mechanisms under the complete and limited information conditions. We first examine the level of convergence to Nash equilibrium by checking the proportion of equilibrium play as well as the distance from Nash equilibria. We then investigate the speed of convergence to Nash equilibrium. Lastly, we examine the efficiency under each treatment.

[Figures 1 and 2 about here.]

Figures 1 and 2 present the experimental data under complete and limited information respectively. In each figure, the top four panels present the time series mean strategies (dots), standard deviation (error bars) and equilibrium values (dashed lines) of each of the four types averaged across five independent sessions under ACP. The bottom four panels present the same information under SRL. Note that in the ACP panels, multiple equilibria correspond to two dashed lines representing the upper and lower bound of the equilibrium values. Comparing the top with the bottom four panels, it seems that SRL converges to equilibrium much faster than ACP in both figures. Another important feature is that convergence seems much faster under complete information. In what follows, we will present statistical analysis of these patterns.

We use the proportion of Nash equilibrium play, $P^{e}$, as a measure for convergence level. We use the point prediction of $(6,7,8,9)$ for the SRL mechanism, and a set prediction of $(\{3,4,5\},\{9,10,11\}$, $\{13,14,15\},\{15,16,17\})$ for the ACP mechanism. As this measure punishes all non-equilibrium choices equally, we use the distance from equilibrium, ${ }^{9} D_{i, t}^{e} \equiv\left|q_{i}^{t}-q_{i}^{e}\right|$, as a second measure of convergence level, which rewards choices close to equilibrium.

RESULT 1 (Equilibrium Play: Comparison of Mechanisms) Under complete information, the proportion of Nash equilibrium play (distance from equilibrium) under SRL is significantly higher (lower) than that under ACP. Under limited information, the proportion of Nash equilibrium play (distance from equilibrium) under SRL is weakly higher (significantly lower) than that under ACP.

\section{[Table 4 about here.]}

\footnotetext{
${ }^{8}$ Note that in this experimental setting the maximum possible aggregate payoff is 881 at strategy four-tuple $(0,0,9,20)$, which is obtained through an exhaustive grid search over the entire strategy space.

${ }^{9}$ Using alternative measures, such as the mean squared difference of choices from equilibrium, does not change the main results.
} 
SUPPORT: Table 4 presents the proportion of Nash equilibrium play and the average distance from equilibrium for each session. We use $P^{e}$ to denote the proportion of equilibrium play. Permutation tests (onetailed) reject the null hypothesis that the proportion of Nash equilibrium play under SRL is the same as that under ACP in favor of the alternative hypotheses that $P^{e}\left(S R L_{c}\right)>P^{e}\left(A C P_{c}\right)$ at $p=0.004$, and that $P^{e}\left(S R L_{l}\right)>P^{e}\left(A C P_{l}\right)$ at $p=0.083$. The corresponding one-tailed Wilcoxon rank sum tests reject the null hypotheses of equal distance from equilibrium in favor of the alternative hypotheses that $D^{e}\left(S R L_{c}\right)>D^{e}\left(A C P_{c}\right)$ at $p=0.009$ and $D^{e}\left(S R L_{l}\right)>D^{e}\left(A C P_{l}\right)$ at $p=0.016$.

Result 1 indicates that the proportion of Nash equilibrium play is higher under SRL than that under ACP under both information conditions. This result rejects Hypotheses 1 and 2. This result is also in contrast to Chen (2003), where she finds that, in a two-type environment, the performance of the two mechanisms is statistically indistinguishable under complete information, though SRL performs significantly better than ACP under limited information. Our interpretation is that, with two types, the mechanisms can be presented to the subjects as a bi-matrix game, where finding Nash equilibrium is relatively easy. With four types, the bi-matrix game representation is no longer feasible. As a result, the uniform dominance (or overwhelming) property of the SRL game helps subjects to get to equilibrium even under complete information.

RESULT 2 (Equilibrium Play: Comparison of Information Conditions) For both the SRL and ACP mechanisms, the proportion of equilibrium play (distance from equilibrium) under complete information is significantly higher (lower) than that under limited information.

SUPPORT: Using the session level statistics in Table 4, one-tailed permutation tests reject the null hypotheses of equal proportion in favor of the alternative hypotheses that $P^{e}\left(S R L_{c}\right)>P^{e}\left(S R L_{l}\right)$ at $p=0.004$, and that $P^{e}\left(A C P_{c}\right)>P^{e}\left(A C P_{l}\right)$ at $p=0.004$. The corresponding one-tailed Wilcoxon rank sum tests reject the null hypotheses of equal distance from equilibrium in favor of the alternative hypotheses that $D^{e}\left(S R L_{c}\right)>D^{e}\left(S R L_{l}\right)$ at $p=0.009$, and that $D^{e}\left(A C P_{c}\right)>D^{e}\left(A C P_{l}\right)$ at $p=0.016$.

Result 2 indicates that the amount of information significantly impacts the convergence level, possibly through its influence on convergence speed. We are interested in the speed of convergence both at the aggregate mechanism level and at the individual level, as the latter might provide an explanation for the dynamics we observe.

To investigate convergence speed and various factors affecting it, we use a random-effects GLS model, where each group consists of all quantities submitted by one individual. Results of the estimation are reported in Table 5. In six different specifications (columns (1) to (6)), the dependent variable is $D_{i, t}^{e}$, the distance from equilibrium for the individual player. Again, we use the equilibrium point prediction for SRL 
and the set prediction for ACP. In specifications (1) and (3), we use $\ln ($ Period) as the independent variable to investigate whether Period (or time) has a significant effect on convergence speed (Chen and Gazzale 2004). To examine whether the effects of learning remain constant, decreasing or increasing over time, we used Period, $\ln$ (Period), as well as Period ${ }^{2}$ as independent variables. Since specifications with $\ln ($ Period) overall yields the best fit, we report only these specifications. In specifications (2) and (4), we add a dummy variable for the information conditions, CompleteInfo, which is equal to one for complete information and zero for limited information. The interaction of CompleteInfo and $\ln ($ Period) captures the effects of more information on convergence speed. In specifications (5) and (6), we add a mechanism dummy, SRL, which is equal to one for SRL and zero for ACP. Compared with the coefficient of $\ln$ (Period), the coefficient for the interaction term, $\mathrm{SRL} \times \ln ($ Period), captures the difference between SRL and ACP on convergence speed.

\section{RESULT 3 (Convergence Speed: Information and Mechanism Effects) Convergence to equilibrium sig-} nificantly increases over time. More information significantly increases convergence speed for ACP. Under both information conditions, convergence is significantly more rapid under SRL.

\section{[Table 5 about here.]}

SUPPORT: Table 5 reports results of random-effects GLS regressions. In specifications (1) and (3), the coefficients of $\ln$ (Period) are both negative and highly significant, indicating increased convergence over time. In specifications (2) and (4), the coefficients for CompleteInfo $\times \ln ($ Period) are both negative, but only significant under ACP. In specifications (5) and (6), the coefficients for SRL $\times \ln ($ Period) are both negative and highly significant, indicating more rapid convergence under SRL than under ACP.

The first part of Result 3 indicates that players learn to play equilibrium strategies over time, which is not surprising. The second part indicates that more information increases the speed of convergence, but this information effect is only significant for ACP, not for SRL. Unlike ACP, the speed of convergence under SRL does not depend critically on the amount of information players have about the underlying structure of the game. Therefore, if the SRL mechanism is used in limited information settings, such as the Internet, we expect the same convergence speed as in complete information settings. By Result 3, we reject Hypotheses 3 and 4.

We now examine whether there exist type specific effects on the level and speed of convergence. We use a random-effects GLS model, where the dependent variable is again $D_{i, t}^{e}$, the distance between actual quantity demanded and equilibrium quantity. In each of the four specifications, the independent variables are the type dummies (Type $i$, where $i=2,3,4), \ln$ (Period), and interactions of type dummies and $\ln ($ Period). 
The omitted dummy variable is Type 1 . Therefore, the Constant measures the convergence level of Type 1, while the coefficient of Type $i$ measures the difference in convergence level between Type $i$ and Type 1 . Similarly, the coefficient of $\ln$ (Period) measures the convergence speed for Type 1, while the coefficient of Type $i \times \ln$ (Period) measures the difference in convergence speed between Type $i$ and Type 1 . Results of the estimation are reported in Table 6.

\section{[Table 6 about here.]}

Under SRL, while the convergence levels, as captured by the coefficients of type dummies, do not differ significantly across types at the conventional level, ${ }^{10}$ convergence speed does.

RESULT 4 (Convergence Speed by Type under SRL) Under SRL complete information, convergence speed follows the order of Type $1 \sim$ Type $2 \sim$ Type $4>$ Type 3. Under SRL limited information, convergence speed follows the order of Type $1>$ Type $2>$ Type $3 \sim$ Type 4 .

SUPPORT: Specification (1) and (2) in Table 6 reports results of estimation under SRL complete and limited information respectively. Coefficient of $\ln ($ Period), and coefficient of $\ln ($ Period $)+$ coefficient of Type $i \times \ln ($ Period) measures the convergence speed of Type 1 and Type $i$ respectively. The more negative a coefficient is, the faster the convergence speed is.

Result 4 reveals how individual learning takes place under SRL and why it converges so robustly under both complete and limited information conditions. Recall that under SRL a player's cost share is independent of demands higher than her own. Therefore, once users 1 to $i$ find the equilibrium quantities and settle down, the $(i+1)^{t h}$ user's problem becomes simple. Under complete information, some players might be able to find the equilibrium quantities through various degrees of introspection without waiting for smaller users to settle down first. Therefore, while the order of settling down helps the speed of convergence, it is not crucial. We do observe that Type 1,2 and Type 4 have statistically indistinguishable speeds of convergence. In limited information settings, however, this order of settling down becomes especially important, as rational introspection is not feasible, while experimentation and hill-climbing are the key elements of learning. The second part of Result 4 indicates that the speed of convergence by type follows essentially the same order of settling down analyzed before, with the speed of Types 3 and 4 statistically indistinguishable $(p>0.10)$.

Under ACP, the Type 1 convergence level is significantly lower than those of the other types under both complete and limited information. This pattern is also confirmed from Figures 1 and 2. The convergence

\footnotetext{
${ }^{10}$ We use a 5\% statistical significance level to claim existence of any causal effects. Note that under SRL, convergence levels for types 2 and 3 are weakly higher $(p<0.10)$ than those for types 1 and 4 under complete information.
} 
speed, on the other hand, does not exhibit any type-specific pattern as in SRL.

RESULT 5 (Convergence Speed by Type under ACP) Under ACP complete information, the convergence speed follows the order of Type $1>$ Type $4>$ Type $3>$ Type 2. Under ACP limited information, however, convergence speed is not significantly different across types.

SUPPORT: Specifications (3) and (4) in Table 6 reports results of estimation under ACP complete and limited information respectively. Again, coefficient of $\ln ($ Period), and coefficient of $\ln ($ Period $)+$ coefficient of Type $i \times \ln ($ Period) measures Types 1 and $i$ 's convergence speed respectively. The more negative a coefficient is, the faster the convergence speed is.

As discussed in Section 2, under ACP, a player's cost share is affected by everyone else's demand, thus, we do not expect a clear order of settling down by type. With complete information, various degrees of rational introspection might help convergence to equilibrium. Under limited information, however, as one player's experimentation immediately affects every other player's payoff, this makes learning difficult. Result 5 confirms this observation.

We now compare the efficiency of the mechanisms. Let $E f$ denote efficiency.

[Table 7 about here.]

RESULT 6 (Efficiency: Comparison of the Two Mechanisms) The efficiency of the SRL mechanism is significantly higher than that of the ACP mechanism under both the complete and the limited information treatments.

SUPPORT: Table 7 reports the efficiency of each session under each treatment. Permutation tests rejects the null hypotheses of equal efficiency in favor of the alternative hypotheses that $\operatorname{Ef}\left(\mathrm{SRL}_{c}\right)>\operatorname{Ef}\left(\mathrm{ACP}_{c}\right)$ at a significance level of 0.004 (one-tailed); $\operatorname{Ef}\left(\mathrm{SRL}_{l}\right)>\operatorname{Ef}\left(\mathrm{ACP}_{l}\right)$ at a significance level of 0.004 (one-tailed); and $\operatorname{Ef}\left(\mathrm{SRL}_{l}\right)>\operatorname{Ef}\left(\mathrm{ACP}_{c}\right)$ at a significance level of 0.004 (one-tailed).

Result 6 indicates that the SRL mechanism performs robustly better than the ACP mechanism in terms of group efficiency regardless of information conditions. The efficiency of the SRL mechanism under the limited information treatment is significantly higher than the ACP mechanism under the complete information condition. This result is consistent with Results 1 and 3. Next, we compare the efficiency within each mechanism under different information conditions.

RESULT 7 (Efficiency: Comparison of Information Conditions) For both the SRL and ACP mechanisms, the efficiency under complete information is significantly higher than that under limited information. 
SUPPORT: Table 7 reports the efficiency of each independent observation under each treatment. Permutation tests reject the null hypotheses of equal efficiency in favor of the alternative hypotheses that $\operatorname{Ef}\left(\operatorname{SRL}_{c}\right)$ $>\operatorname{Ef}\left(\mathrm{SRL}_{l}\right)$ at a significance level of 0.004 (one-tailed), and that $\operatorname{Ef}\left(\mathrm{ACP}_{c}\right)>\operatorname{Ef}\left(\mathrm{ACP}_{l}\right)$ at a significance level of 0.048 (one-tailed).

Result 7 indicates that more information is advantageous for aggregate efficiency, which is consistent with Result 2.

Experimental results indicate that under both complete and limited information settings SRL performs robustly better than ACP in terms of proportion of Nash equilibrium play, convergence speed and efficiency. The property that a user's cost share is independent of larger users implies an order (sorted by the quantity demanded) of settling down to equilibrium strategies under SRL, which facilitates learning and convergence especially under limited information settings. The uniform dominance property of SRL also implies that sampling and experimentation are much more informative than that under ACP.

To check robustness of the experimental results in different environments, we conducted Monte Carlo simulations using a payoff-assessment learning model (Sarin and Vahid 1999) (Kirman and Vriend 2001), calibrated against experimental data. We then use the calibrated parameters to simulate the dynamic paths in nine different environments. Simulation results indicate that, while the experimental results hold when preferences exhibit decreasing or constant returns, the performance of the two mechanisms is similar with increasing returns. Simulation results are not reported here due to space limitations, but are available from the authors upon request.

\section{Conclusion}

Cost sharing mechanisms have many practical applications in the real world. An increasingly important area is distributed systems like the Internet, where agents have very limited information about the payoff structure as well as the characteristics of other agents. Most current Internet routers use the FIFO or average cost pricing mechanism, while this study suggests that the fair queuing or serial mechanism might be a better choice.

This paper reports experimental results on the serial and the average cost pricing mechanisms under two information conditions. The first is a complete information condition designed to test the basic properties of the mechanisms. The other simulates distributed systems by giving the subjects very limited information about the game. The latter present a more challenging and realistic setting for the cost sharing mechanisms. Experimental results show that the serial mechanism performs significantly better than the average cost 
pricing mechanism in all treatments in terms of efficiency and predictability measured as frequency of equilibrium play, as well as the speed of convergence.

\section{APPENDIX. Discretization and Multiple Equilibria in ACP}

Proof of Proposition 1: Let $\left\{q_{i}^{*}\right\}_{i}$ be the Nash equilibrium quantities of the ACP game with a continuous strategy space. With a quadratic cost function, $C\left(\sum_{i} q_{i}\right)$, the unique Nash equilibrium is characterized by the solution to the following maximization problem:

$$
\max _{q_{i}} \alpha_{i} q_{i}-\frac{q_{i}}{\sum_{j} q_{j}}\left(\sum_{j} q_{j}\right)^{2}
$$

The first order condition is $\alpha_{i}-\sum_{j} q_{j}-q_{i}=0$. Summing over $i$, we get $\sum_{i} q_{i}=\sum_{i} \alpha_{i} /(n+1)$. Therefore,

$$
q_{i}^{*}=\alpha_{i}-\frac{\sum_{j} \alpha_{j}}{n+1}, \sum_{i} q_{i}^{*}=\frac{\sum_{i} \alpha_{i}}{n+1}, \text { and } \pi_{i}^{*}=\left(q_{i}^{*}\right)^{2} .
$$

To prove that $\left\{\bar{q}_{1}, \cdots, \bar{q}_{n} \mid \bar{q}_{i} \in\left\{q_{i}^{*}-s, q_{i}^{*}, q_{i}^{*}+s\right\}\right.$ and $\left.\sum_{i} \bar{q}_{i}=\sum_{i} q_{i}^{*}\right\}$ are all Nash equilibria of the discrete game, we need to show that unilateral defection by any player does not improve her payoff. In equilibrium

$$
\bar{\pi}_{i}(\bar{q})=\alpha_{i} \bar{q}_{i}-\bar{q}_{i} \sum_{j} \bar{q}_{j}=\alpha_{i} \bar{q}_{i}-\bar{q}_{i} \sum_{j} q_{j}^{*}=\bar{q}_{i}\left(\alpha_{i}-\frac{\sum_{i} \alpha_{i}}{n+1}\right)=\bar{q}_{i} q_{i}^{*} .
$$

Case 1. $\bar{q}_{i}=q_{i}^{*}-s$. In this case $\bar{\pi}_{i}(\bar{q})=\left(q_{i}^{*}-s\right) q_{i}^{*}$.

If player $i$ unilaterally defects to strategy $q_{i}=q_{i}^{*}-m \equiv \bar{q}_{i}+s-m$, where $m \in D$ and $m \neq s$, $\pi_{i}\left(q_{i}, \bar{q}_{-i}\right)=\left(q_{i}^{*}-m\right)\left[\alpha_{i}-\left(\sum_{j} q_{j}^{*}+s-m\right)\right]=\left(q_{i}^{*}-m\right)\left(q_{i}^{*}+m-s\right)=\left(q_{i}^{*}-s\right) q_{i}^{*}-m(m-s) \leq\left(q_{i}^{*}-s\right) q_{i}^{*}$, since $m(m-s) \geq 0$ for $m \in D$.

Case 2. $\bar{q}_{i}=q_{i}^{*}$. In this case $\bar{\pi}_{i}(\bar{q})=\left(q_{i}^{*}\right)^{2}$.

If player $i$ unilaterally defects to strategy $q_{i}=q_{i}^{*}+m \equiv \bar{q}_{i}+m$, where $m \in D$ and $m \neq 0, \pi_{i}\left(q_{i}, \bar{q}_{-i}\right)=$ $\left(q_{i}^{*}+m\right)\left[\alpha_{i}-\left(\sum_{j} q_{j}^{*}+m\right)\right]=\left(q_{i}^{*}+m\right)\left(q_{i}^{*}-m\right)=\left(q_{i}^{*}\right)^{2}-m^{2}<\left(q_{i}^{*}\right)^{2}$.

Case 3. $\bar{q}_{i}=q_{i}^{*}+s$. In this case $\bar{\pi}_{i}(\bar{q})=\left(q_{i}^{*}+s\right) q_{i}^{*}$.

If player $i$ unilaterally defects to strategy $q_{i}=q_{i}^{*}+m \equiv \bar{q}_{i}-s+m$, where $m \in D$ and $m \neq s$, $\pi_{i}\left(q_{i}, \bar{q}_{-i}\right)=\left(q_{i}^{*}+m\right)\left[\alpha_{i}-\left(\sum_{j} q_{j}^{*}-s+m\right)\right]=\left(q_{i}^{*}+m\right)\left(q_{i}^{*}-m+s\right)=\left(q_{i}^{*}+s\right) q_{i}^{*}-m(m-s) \leq\left(q_{i}^{*}+s\right) q_{i}^{*}$, since $m(m-s) \geq 0$ for $m \in D$.

Therefore, $\left\{\bar{q}_{1}, \cdots, \bar{q}_{n} \mid \bar{q}_{i} \in\left\{q_{i}^{*}-s, q_{i}^{*}, q_{i}^{*}+s\right\}\right.$ and $\left.\sum_{i} \bar{q}_{i}=\sum_{i} q_{i}^{*}\right\}$ are all Nash equilibria of the discrete game.

Let $\bar{q}_{1} \leq \bar{q}_{2} \leq \cdots \leq \bar{q}_{n}$. Let $\bar{q}_{i}=q_{i}^{*}+s_{i}$, where $s_{i}=-s, 0$ or $s$ and $\sum_{i} s_{i}=0$. The aggregate payoff in equilibrium is 


$$
\sum_{i} \pi_{i}\left(\bar{q}_{i}\right)=\sum_{i} \bar{q}_{i} q_{i}^{*}=\sum_{i}\left(q_{i}^{*}\right)^{2}+\sum_{i} s_{i} q_{i}^{*} .
$$

We now show that there are no other equilibria in pure strategies. The argument is in two cases, both established by contradiction.

Case A. To show there are no other equilibria in which $\sum_{i} \bar{q}_{i}=\sum_{i} q_{i}^{\star}$, suppose that for player $i$, $\bar{q}_{i}=q_{i}^{\star}+k s$ for $k \geq 2$. Then, it must be that $\sum_{j \neq i} \bar{q}_{j}=\sum_{j \neq i} q_{i}^{\star}-k s$. But inspection of the reaction function shows that for every $k s$ the quantity chosen by players other than $i$ is decreased, the best reply for player $i$ increases by $\frac{k s}{2}$. Therefore, $q_{i}^{\star}+(k-1) s$ is strictly better for player $i$ than $q_{i}^{\star}+k s$, and so this cannot be an equilibrium. The symmetric argument covers the case of $\bar{q}_{i}=q_{i}^{\star}-k s$ for $k \geq 2$.

Case B. To show that there can be no equilibrium in which $\sum_{i} \bar{q}_{i} \Leftarrow \sum_{i} q_{i}^{\star}$, write $\bar{q}_{i}=q_{i}^{\star}+k_{i} s$ for all $i$; then $\left|\sum_{j} k_{j} s\right| \geq 1$ by assumption. The best reply for each player $i$, not restricted to the discrete grid, is to choose

$$
q_{i}^{\mathrm{BR}}=\frac{1}{2}\left[\alpha_{i}-\sum_{j \neq i} q_{j}\right]=\frac{1}{2}\left[\alpha_{i}-\sum_{j \neq i} q_{i}^{\star}-\sum_{j \neq i} k_{j} s\right] .
$$

Note that if the $\bar{q}_{i}$ form an equilibrium, then $\left|\bar{q}_{i}-q_{i}^{\mathrm{BR}}\right| \leq \frac{s}{2}$. Summing over all players and manipulating gives

$$
\begin{aligned}
\sum_{j} q_{j}^{\mathrm{BR}} & =\frac{1}{2}\left[\sum_{j} \alpha_{j}-(n-1) \sum_{j} q_{j}^{\star}-(n-1) \sum_{j} k_{j} s\right] \\
& =\frac{1}{2}\left[(n+1) \sum_{j} q_{j}^{\star}-(n-1) \sum_{j} q_{j}^{\star}-(n-1) \sum_{j} k_{j} s\right] \\
& =\sum_{j} q_{j}^{\star}-(n-1) \sum_{j} k_{j} s \\
\sum_{j} q_{j}^{\mathrm{BR}}-\sum_{j} \bar{q}_{j} & =\sum_{j} q_{j}^{\star}-(n-1) \sum_{j} k_{j} s-\sum_{j} \bar{q}_{j} \\
& =\sum_{j} q_{j}^{\star}-(n-1) \sum_{j} k_{j} s-\sum_{j}\left(q_{j}^{\star}+k_{j} s\right) \\
& =\frac{n+1}{2} \sum_{j} k_{j} s .
\end{aligned}
$$

However, $\left|\sum_{j} q_{j}^{\mathrm{BR}}-\sum_{j} \bar{q}_{j}\right| \leq \frac{n}{2} s$ and $\left|\frac{n+1}{2} \sum_{j} k_{j} s\right| \geq \frac{n+1}{2} s$, so this last equation cannot hold, and the contradiction is established.

Proof of Proposition 2: Begin by assuming w.l.o.g. that the possible quantities $q_{i}$ are integers; if not, change units. Further assume that the unique equilibrium obtained by elimination of dominated strategies in the continuous game is in integers. By inspection of the payoff function, the $\alpha_{i}$ must be integers in order 
for player $i$ to be indifferent between two quantities. The payoff function for each player $i$ is quadratic in $q_{i}$, holding fixed the other players' behavior. Therefore, if two quantities $q_{i}<q_{i}^{\prime}$ are both best responses, it must be that $q_{i}^{\prime}=q_{i}+1$, and that the unrestricted best response is $q_{i}+\frac{1}{2}$. Let $\mathcal{I}$ be the set of indices of players who are indifferent in equilibrium between two such quantities; each player $i \in \mathcal{I}$ plays $q_{i}+1$ with probability $p_{i}$ and $q_{i}$ with probability $1-p_{i}$. All other players $i \in \mathcal{E}$ play their strict best reply quantity $q_{i}$ with probability one. For these players $i \notin \in \mathcal{I}$,

$$
q_{i}=\frac{1}{2}\left[a_{i}-\sum_{j \neq i} q_{j}-\sum_{j \in \mathcal{I}} p_{j}\right],
$$

which can be rearranged to

$$
\sum_{j \in \mathcal{I}} p_{j}=\alpha_{i}-\sum_{j} q_{j}-q_{i}
$$

For players $i \in \mathcal{I}$, the best-reply condition is

$$
q_{i}+\frac{1}{2}=\frac{1}{2}\left[a_{i}-\sum_{j \neq i} q_{j}-\sum_{j \in \mathcal{I}, j \neq i} p_{j}\right]
$$

which rearranges to

$$
\sum_{j \in \mathcal{I}, j \neq i} p_{j}=\alpha_{i}-1-\sum_{j} q_{j}-q_{i} .
$$

The argument proceeds in three steps, each of which is established by contradiction.

Step 1. No equilibrium in which some players randomize and others have a strict best reply.

If there is some player $j \notin \mathcal{I}$ and $i \in \mathcal{I}$, then subtracting (2) from (1) gives

$$
p_{i}=\alpha_{j}-\alpha_{i}-1-q_{j}+q_{i}
$$

Since the right side is an integer, $p_{i} \in\{0,1\}$, and thus player $i$ does not randomize. Therefore, if there are any equilibria in which players randomize, it must be that all players are indifferent between two quantities $q_{i}$ and $q_{i}+1$, and have a best reply satisfying (2). That is to say, all players are in $\mathcal{I}$, but it remains possible that some actively randomize while others play a pure strategy which is only a weak best reply.

Step 2. No equilibrium in which all players randomize.

For any two players $i$ and $k$, equation (2) implies by subtraction

$$
p_{i}-p_{k}=\alpha_{i}-\alpha_{k}-q_{i}+q_{k}
$$

Since the right side is an integer, $p_{i}-p_{k}$ is an integer. If both players actively randomize, the only way for this to hold is for $p_{i}-p_{k}=0$, or $p_{i}=p_{k}$. Since this is true for any $i$ and $k$, it must be that all the $p_{i}$ are 
identical; let $p \equiv p_{i}$ for all $i$. Then (2) becomes

$$
(n-1) p=\alpha_{i}-1-\sum_{j} q_{j}-q_{i}
$$

Since the right side of this is an integer, it must be that $p=\frac{K}{n-1}$ for some $K \in\{1,2, \cdots, n-2\}$. With this substitution, summing (3) over all players then gives

$$
n K=\sum_{j} \alpha_{j}-n-(n+1) \sum_{j} q_{j}
$$

or

$$
\sum_{j} q_{j}=\frac{\sum_{j} \alpha_{j}-n[K+1]}{n+1} .
$$

The left side of this must be an integer. While $\frac{\sum_{j} \alpha_{j}}{n+1}$ is an integer by assumption, since it is the total quantity in the unique equilibrium in the continuous version, $\frac{n[K+1]}{n+1}$ cannot be, since $0<K<n+1$ by definition; therefore, there is a contradiction.

\section{Step 3. No equilibrium in which more than one player randomizes.}

From Step 1, it is already known that all players must be indifferent between two quantities $q_{i}$ and $q_{i}+1$. Of these players, let $\mathcal{H}$ be the set of players who play the larger quantity of the two over which they are indifferent, $\mathcal{L}$ be the set of players playing the lower of the quantities, and $\mathcal{R}$ being the set who randomize. For players $i \in \mathcal{H}$, the best-reply condition implies

$$
\sum_{j \in \mathcal{R}, j \neq i} p_{j}=\alpha_{i}+1-\sum_{j} q_{j}-q_{i}
$$

The same argument from Step 2 applies to show that all players who randomize do so with the same probability $p$ on the higher quantity, and that $p=\frac{K}{n-1}$ for some $K \in\{1,2, \cdots,|\mathcal{R}|-2\}$. Summing over all players gives

$$
\begin{aligned}
(|\mathcal{R}|-1) p & =\sum_{j} \alpha_{j}+(|\mathcal{H}|-|\mathcal{R}|-|\mathcal{L}|)-(n+1) \sum_{j} q_{j} \\
\sum_{j} q_{j} & =\frac{\sum_{j} \alpha_{j}+K-(|\mathcal{H}|-|\mathcal{R}|-|\mathcal{L}|)}{n+1}
\end{aligned}
$$

Since $|\mathcal{H}|+|\mathcal{R}|+|\mathcal{L}|=n$, and $0<k<|\mathcal{R}|-1$, the right side cannot be an integer, and thus there is a contradiction. 
Table 1: Features of Experimental Treatments

\begin{tabular}{ccc}
\hline \hline & \multicolumn{2}{c}{ Information Conditions } \\
\hline & Complete Information & Limited Information \\
\hline $\mathrm{SRL}$ & $\mathrm{SRL}_{c}$ & $\mathrm{SRL}_{l}$ \\
& $(5$ sessions $)$ & $(5$ sessions $)$ \\
\hline $\mathrm{ACP}$ & $\mathrm{ACP}_{c}$ & $\mathrm{ACP}_{l}$ \\
& $(5$ sessions $)$ & $(5$ sessions $)$ \\
\hline \hline
\end{tabular}

Table 2: Parameters, Equilibrium Quantities and Payoffs

\begin{tabular}{cl|rrr|rrrr|rr}
\hline \hline \multicolumn{2}{c}{ Subjects } & \multicolumn{3}{c}{ Parameters } & \multicolumn{3}{c}{ Equil. Quantities } & \multicolumn{2}{c}{ Equil. Payoffs } \\
\hline Type & Label & $\alpha_{i}$ & $\omega_{i}^{s}$ & $\omega_{i}^{a}$ & $q_{i}^{s}$ & & $q_{i}^{a}$ & $\pi_{i}^{s}$ & $\pi_{i}^{a}$ \\
\hline 1 & (Blue) & 48 & 60 & 180 & $\mathbf{6}$ & $\{3$, & $\mathbf{4 ,}$ & $5\}$ & $\mathbf{2 0 4}$ & $\mathbf{1 9 6}$ \\
2 & (Green) & 54 & 20 & 102 & $\mathbf{7}$ & $\{9$, & $\mathbf{1 0}$, & $11\}$ & $\mathbf{2 0 3}$ & $\mathbf{2 0 2}$ \\
3 & (Red) & 58 & 0 & 0 & $\mathbf{8}$ & $\{13$, & $\mathbf{1 4}$, & $15\}$ & $\mathbf{2 1 3}$ & $\mathbf{1 9 6}$ \\
4 & (Yellow) & 60 & 0 & 0 & $\mathbf{9}$ & $\{15$, & $\mathbf{1 6}$, & $17\}$ & $\mathbf{2 3 0}$ & $\mathbf{2 5 6}$ \\
\hline \multicolumn{1}{r|}{} & Total & 220 & 80 & 282 & 30 & & 44 & 850 & 850 \\
\hline \hline
\end{tabular}


Table 3: Multiple Equilibrium Quantities and Payoffs in ACP

\begin{tabular}{c|rrrr|rrrr|r}
\hline \hline Number & $q_{1}^{a}$ & $q_{2}^{a}$ & $q_{3}^{a}$ & $q_{4}^{a}$ & $\pi_{1}^{a}$ & $\pi_{2}^{a}$ & $\pi_{3}^{a}$ & $\pi_{4}^{a}$ & $\sum_{i} \pi_{i}^{a}$ \\
\hline 1 & 3 & 9 & 15 & 17 & 192 & 192 & 210 & 272 & 866 \\
2 & 3 & 10 & 14 & 17 & 192 & 202 & 196 & 272 & 862 \\
3 & 3 & 10 & 15 & 16 & 192 & 202 & 210 & 256 & 860 \\
4 & 3 & 11 & 13 & 17 & 192 & 212 & 182 & 272 & 858 \\
5 & 3 & 11 & 14 & 16 & 192 & 212 & 196 & 256 & 856 \\
6 & 3 & 11 & 15 & 15 & 192 & 212 & 210 & 240 & 854 \\
7 & 4 & 9 & 14 & 17 & 196 & 192 & 196 & 272 & 856 \\
8 & 4 & 9 & 15 & 16 & 196 & 192 & 210 & 256 & 854 \\
9 & 4 & 10 & 13 & 17 & 196 & 202 & 182 & 272 & 852 \\
$\mathbf{1 0}$ & $\mathbf{4}$ & $\mathbf{1 0}$ & $\mathbf{1 4}$ & $\mathbf{1 6}$ & $\mathbf{1 9 6}$ & $\mathbf{2 0 2}$ & $\mathbf{1 9 6}$ & $\mathbf{2 5 6}$ & $\mathbf{8 5 0}$ \\
11 & 4 & 10 & 15 & 15 & 196 & 202 & 210 & 240 & 848 \\
12 & 4 & 11 & 13 & 16 & 196 & 212 & 182 & 256 & 846 \\
13 & 4 & 11 & 14 & 15 & 196 & 212 & 196 & 240 & 844 \\
14 & 5 & 9 & 13 & 17 & 200 & 192 & 182 & 272 & 846 \\
15 & 5 & 9 & 14 & 16 & 200 & 192 & 196 & 256 & 844 \\
16 & 5 & 9 & 15 & 15 & 200 & 192 & 210 & 240 & 842 \\
17 & 5 & 10 & 13 & 16 & 200 & 202 & 182 & 256 & 840 \\
18 & 5 & 10 & 14 & 15 & 200 & 202 & 196 & 240 & 838 \\
19 & 5 & 11 & 13 & 15 & 200 & 212 & 182 & 240 & 834 \\
\hline \hline
\end{tabular}


Table 4: Proportion of Equilibrium Play and Distance from Nash Equilibrium for Each Session

\begin{tabular}{|c|c|c|c|c|c|c|c|c|}
\hline \multirow{3}{*}{$\begin{array}{c}\text { Metric } \\
\text { Information } \\
\text { Session }\end{array}$} & \multicolumn{4}{|c|}{ Proportion of NE } & \multicolumn{4}{|c|}{$\sum_{i=1}^{12} \sum_{t=1}^{50}\left|q_{i}^{t}-q_{i}^{e}\right| /(600)$} \\
\hline & \multicolumn{2}{|c|}{ Complete } & \multicolumn{2}{|c|}{ Limited } & \multicolumn{2}{|c|}{ Complete } & \multicolumn{2}{|c|}{ Limited } \\
\hline & $\mathrm{SRL}_{c}$ & $\mathrm{ACP}_{c}$ & $\mathrm{SRL}_{l}$ & $\mathrm{ACP}_{l}$ & $\mathrm{SRL}_{c}$ & $\mathrm{ACP}_{c}$ & $\mathrm{SRL}_{l}$ & $\mathrm{ACP}_{l}$ \\
\hline 1 & 0.629 & 0.215 & 0.272 & 0.145 & 0.861 & 2.592 & 2.458 & 3.850 \\
\hline 2 & 0.418 & 0.228 & 0.180 & 0.160 & 1.040 & 3.143 & 3.578 & 3.392 \\
\hline 3 & 0.405 & 0.263 & 0.137 & 0.162 & 1.123 & 2.710 & 3.082 & 4.042 \\
\hline 4 & 0.440 & 0.197 & 0.193 & 0.140 & 1.222 & 3.412 & 2.650 & 4.438 \\
\hline 5 & 0.583 & 0.265 & 0.175 & 0.183 & 0.652 & 2.152 & 2.887 & 3.707 \\
\hline
\end{tabular}

Table 5: Convergence Speed

\begin{tabular}{|c|c|c|c|c|c|c|}
\hline & \multicolumn{6}{|c|}{ Dependent Variable: Distance from equilibrium, $\left|q_{i}^{t}-q_{i}^{e}\right|$} \\
\hline & \multicolumn{2}{|c|}{ SRL } & \multicolumn{2}{|c|}{$\mathrm{ACP}$} & \multirow{2}{*}{$\begin{array}{l}\text { Complete Info. } \\
\text { (5) }\end{array}$} & \multirow{2}{*}{$\begin{array}{l}\text { Limited Info. } \\
\text { (6) }\end{array}$} \\
\hline & (1) & (2) & (3) & (4) & & \\
\hline \multirow[t]{2}{*}{$\ln ($ Period $)$} & -1.0376 & -1.0225 & -0.6170 & -0.5115 & -0.4997 & -0.7581 \\
\hline & $(0.0357)^{* * *}$ & $(0.0429)^{* * *}$ & $(0.0457)^{* * *}$ & $(0.0552)^{* * *}$ & $(0.0395)^{* * *}$ & $(0.0570)^{* * *}$ \\
\hline \multirow[t]{2}{*}{ CompleteInfo $\times \ln ($ Period $)$} & & -0.0301 & & -0.2110 & & \\
\hline & & $(0.0468)$ & & $(0.0619) * * *$ & & \\
\hline \multirow[t]{2}{*}{$\mathrm{SRL} \times \ln ($ Period $)$} & & & & & -0.2726 & -0.5195 \\
\hline & & & & & $(0.0469) * * *$ & $(0.0604)^{* * *}$ \\
\hline \multirow[t]{2}{*}{ Constant } & 5.1161 & 5.1159 & 5.2243 & 5.2243 & 3.8278 & 6.5111 \\
\hline & $(0.1650)^{* * *}$ & $(0.1444)^{* * *}$ & $(0.1953) * * *$ & $(0.1894) * * *$ & $(0.1433) * * *$ & $(0.1886)^{* * *}$ \\
\hline Observations & 5988 & 5988 & 6000 & 6000 & 5988 & 6000 \\
\hline Number of groups & 120 & 120 & 120 & 120 & 120 & 120 \\
\hline
\end{tabular}

1. Random-effects GLS regressions.

2. Standard errors in parentheses.

3. Significant at: $* * * 1 \%$ level.

4. The program failed to record the last round data in $\mathrm{SRL}_{c}$, session 2. Thus, we have 5988 observations for (1), (2) and (5). 
Table 6: Convergence Speed by Type

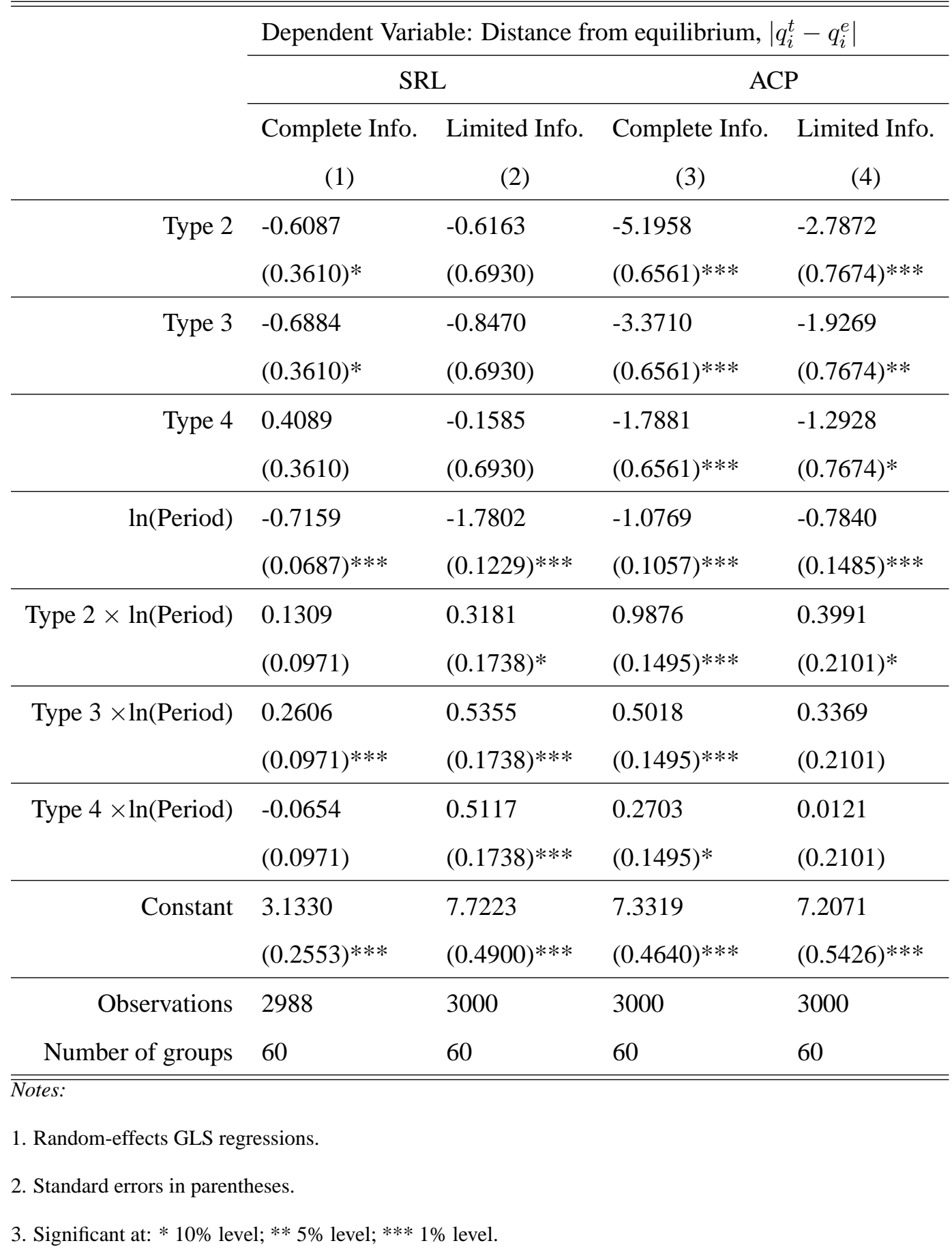


Table 7: Efficiency of Each Session

\begin{tabular}{c|cc|cc}
\hline \hline Information & \multicolumn{2}{|c}{ Complete } & \multicolumn{2}{c}{ Limited } \\
\hline Session & $\mathrm{SRL}_{c}$ & $\mathrm{ACP}_{c}$ & $\mathrm{SRL}_{l}$ & $\mathrm{ACP}_{l}$ \\
\hline 1 & 0.850 & 0.584 & 0.767 & 0.527 \\
2 & 0.861 & 0.614 & 0.733 & 0.628 \\
3 & 0.852 & 0.613 & 0.736 & 0.620 \\
4 & 0.840 & 0.636 & 0.769 & 0.506 \\
5 & 0.871 & 0.662 & 0.763 & 0.492 \\
\hline \hline
\end{tabular}



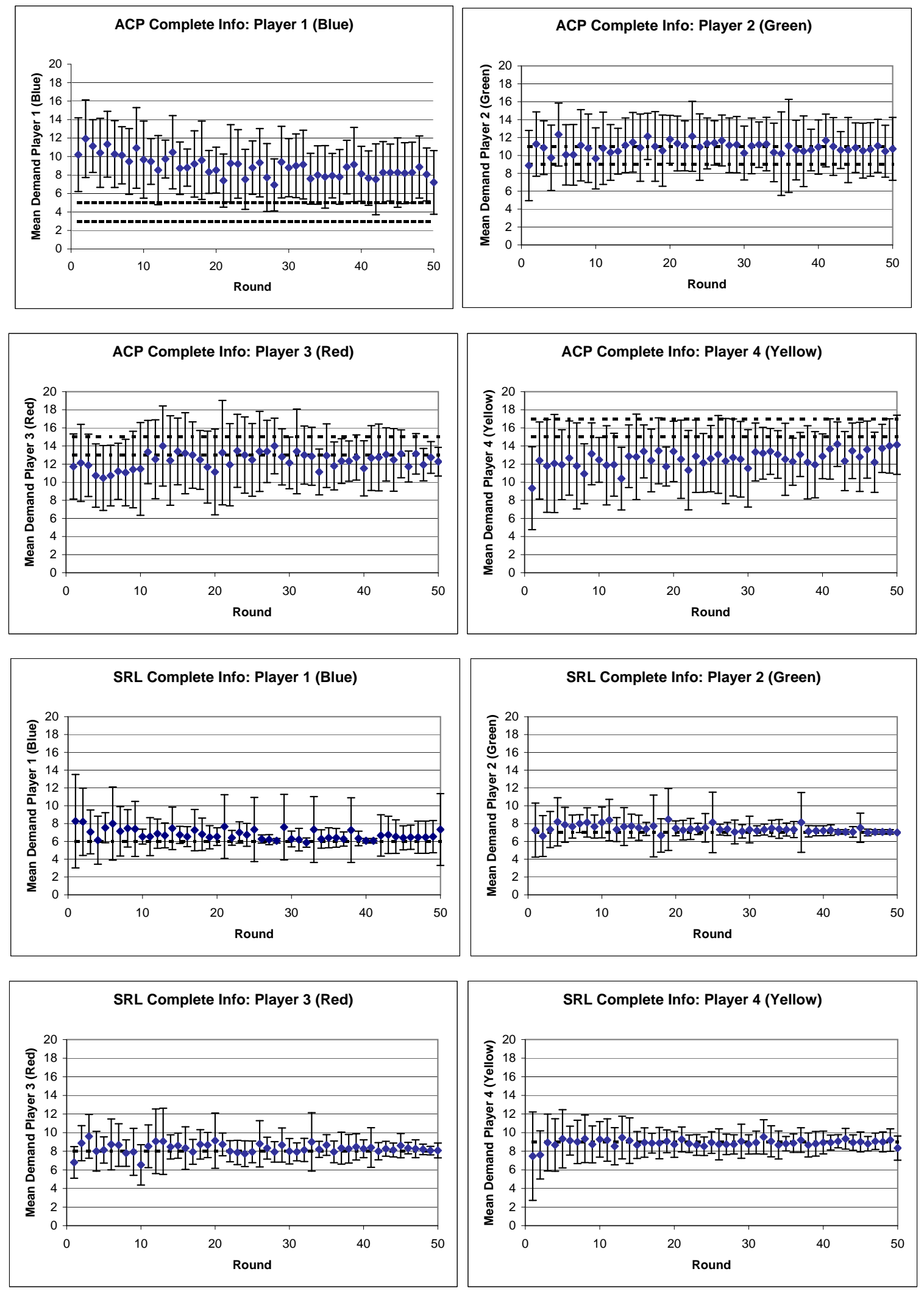

Figure 1: Experimental Data: Complete Information 

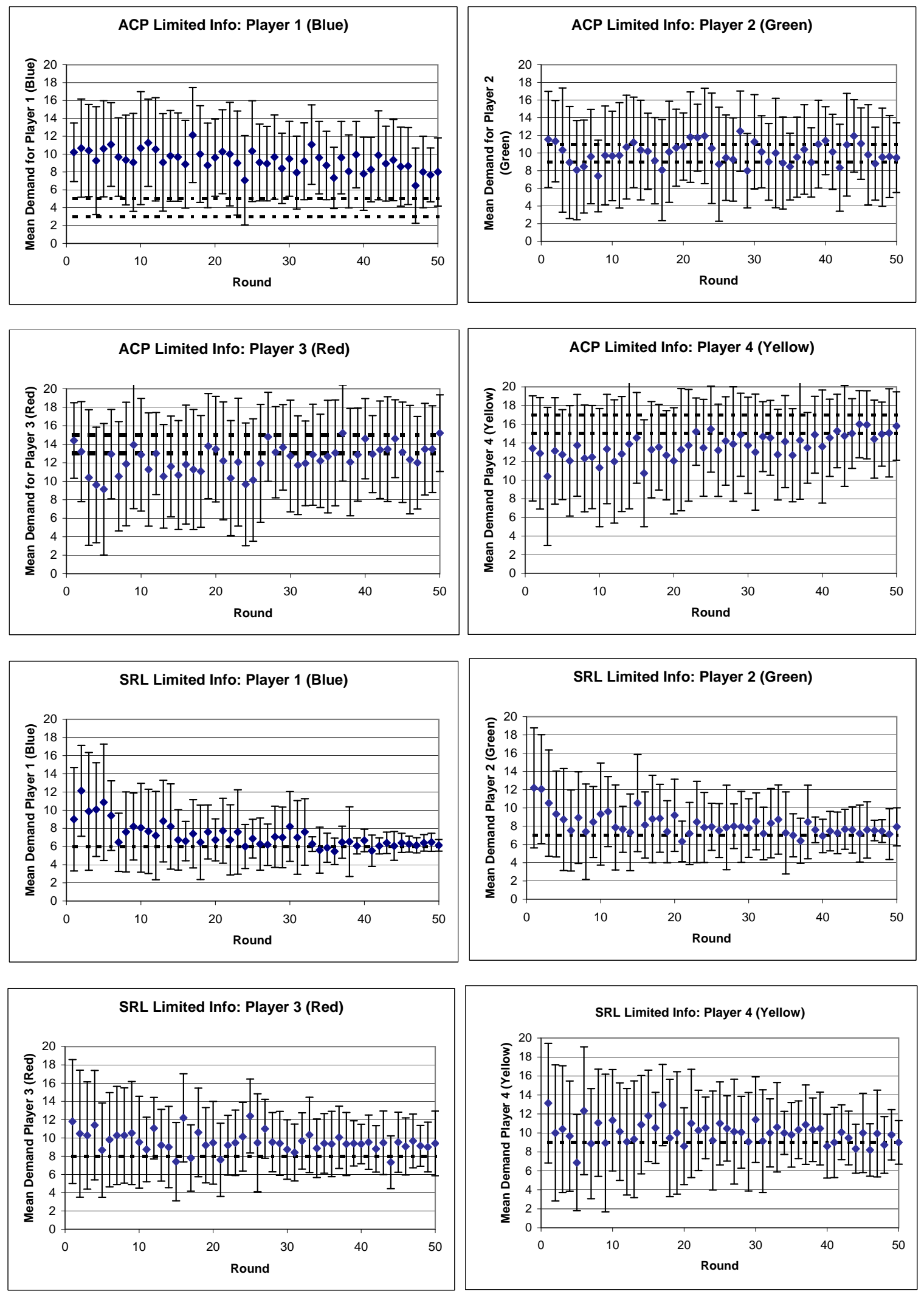

Figure 2: Experimental Data: Limited Information 


\section{References}

Chen, Y.: An experimental study of the serial and average cost pricing mechanisms. J Public Econ, 87, 2305-2335 (2003).

Chen, Y., and Gazzale, R. S.: When does learning in games generates convergence to nash equilibrium? the role of supermodularity in an experimental setting. American Econ Review, 94, 1505-1535 (2004).

Chen, Y., and Khoroshilov, Y.: Learning under limited information. Games and Econ Behavior 44, 1-25 (2003).

Demers, A., S. K., and Shenker, S.: Analysis and simulation of a fair queueing algorithm. J Internetworking 1, 3-26 (1990).

Fischbacher, U.: z-tree: A toolbox for readymade economic experiments - experimenter's manual. University of Zurich Working Paper No. 21 (1999).

Friedman, E., and Moulin, H.: Three methods to share joints costs or surplus. J Econ Theory 87, 275-312 (1999).

Friedman, E., and Shenker, S.: Learning and implementation on the internet. Manuscript: Rutgers University, Department of Economics (1998).

Gailmard, S., and Palfrey, T.: An experimental comparison of collective choice procedures for excludable public goods. J Public Econ 89, 1361-1398 (2005).

Huck, S., and Sarin, R.: Players with limited memory. Contributions to Theoretical Econ 4, Article 6 (2004).

Kirman, A. P., and Vriend, N. J.: Evolving market structure: An ace model of price dispersion and loyalty. J Econ Dynamics \& Control 25, 459-502 (2001).

McKelvey, R. D., McLennan, A. M., and Turocy, T. L.: Gambit: Software tools for game theory, version 0.2006.01.20. 2006. http://econweb. tamu . edu/gambit.

Milgrom, P., and Roberts, J.: Rationalizability, learning and equilibrium in games with strategic complementarities. Econometrica 58, 1255-1277 (1990).

Moulin, H., and Shenker, S.: Serial cost sharing. Econometrica 60, 1009-1037 (1992). 
Moulin, H., and Shenker, S.: Average cost pricing versus serial cost sharing: An axiomatic comparison. J Econ Theory 64, 178-201 (1994).

Rapoport, A., Stein, W., Parco, J., and Seale, D.: Strategic play in single-server queues with endogenously determined arrival times. J Econ Behavior 55, 67-91 (2004).

Razzolini, L., Reksulak, M., and Dorsey, R.: An experimental evaluation of the serial cost sharing rule. Manuscript: University of Mississippi (1999).

Sarin, R., and Vahid, F.: Payoff assessments without probabilities: A simple dynamic model of choice. Games and Econ Behavior 28, 294-309 (1999).

Shenker, S.: Making greed work in networks: A game-theoretic analysis of gateway service disciplines. Sigmetrics, 241-242 (1990).

Stoica, I., Shenker, S., and Zhang, H.: Core-stateless fair queueing: Achieving approximately fair bandwidth allocations in high speed networks. Proceedings of ACM SIGCOMM, 118-130 (1998). 